\title{
Racionalización de mezclas de minerales de hierro para la obtención de sinterizados de calidad optima ${ }^{(\cdot)}$
}

\author{
A. Cores*, S. Ferreira* y M. Muñiz**
}

\begin{abstract}
Resumen
Se utilizaron diversos minerales de hierro, fundentes y coque para preparar cuatro mezclas minerales con distintas proporciones de estos minerales. Con cada mezcla se fabricó una serie de sinterizados en planta piloto. Se caracteriza cada uno de los sinterizados mediante análisis químico y granulométrico, ensayo de la degradación del sinterizado durante la reducción en el horno alto (ensayo RDI), ensayo de la resistencia en frío (ensayo Tumbler), ensayo de reductibilidad; determinación de las temperaturas de reblandecimiento y fusión y determinación de la estructura del sinterizado por microscopia electrónica. Se establecen las condiciones de las mezclas y los parámetros de operación para la obtención de sinterizados de calidad óptima.
\end{abstract}

\section{Rationalisation of iron ore mixtures for obtaining optimum quality sinters}

Abstract

Keywords

\begin{abstract}
Various iron ores, fluxes and coke, were used to prepare four ore mixtures with different proportions of these minerals. With each mixtures a series of sinters were produced in the pilot plant. Each one of the sinters was characterised by: chemical and granulometric analysis; degradation test on the sinter during reduction in the blast furnace, (RDI test); cold resistance test, (Tumbler Test); reducibility test; determining of softening and melting temperatures; and the determination of the structure of the sinter by electron microscopy. T he conditions of the mixtures and the operational parameters for obtaining the optimum quality of the sinters were established.
\end{abstract}

Sintering; Ore mixtures; Iron ores; Blast furnace.

\section{INTRODUCCIÓN}

El proceso de sinterización se utiliza para aglomerar una mezcla de minerales de hierro, fundentes y coque, de tamaño de partícula inferior a $8 \mathrm{~mm}$, de tal forma que el sinterizado producido, con tamaño tamizado entre 12 y $35 \mathrm{~mm}$, una vez cargado en el hormo alto, puede soportar las presiones y temperaturas del horno.

En el proceso de sinterización, la mezcla de mineral se somete, previamente, a una granulación que consiste en homogenizar la mezcla en un tambor giratorio, con la adición de un 6-8 \% de agua, durante unos minutos.

Los gránulos formados se cargan sobre la parilla de la banda de sinterización, en donde la temperatura de los gránulos se eleva entre 1.250 y $1.350^{\circ} \mathrm{C}$ para producir la fusión parcial y producir material semifundido, el cual, durante el enfriamiento posterior, cristaliza en varias fases minerales de distinta composición química y morfológica, principalmente, hematita, magnetita, ferritos y ganga formada en sus mayor parte por silicatos cálcicos ${ }^{[1-3]}$. La energía del proceso la suministra la combustión del coque.

Los factores que afectan a la sinterización y a la calidad del sinterizado producido son, entre otros: a) el tamaño y composición de los gránulos; b) el tamaño, composición y propiedades relativas de los componentes de la alimentación (minerales de hierro, fundentes y coque); c) la composición mineralógica

(•) Trabajo recibido el día 7 de agosto de 2008 y aceptado en su forma final el día 7 de enero de 2009.

* Centro Nacional de Investigaciones Metalúrgicas (CENIM-CSIC) Avda. Gregorio del Amo, 8, 28040 Madrid, España. alcores@cenim.csic.es, serafin@cenim.csic.es.

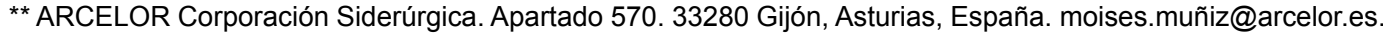


de los diferentes componentes de la alimentación y d) el perfil térmico del proceso.

El presente trabajo de investigación tiene como objetivos optimizar las condiciones de marcha en la sinterización y fabricar un sínter de la mejor calidad. Para ello, se ha realizado una caracterización extensa de los minerales y fundentes y, después, se ha procedido a la elaboración de unas leyes racionales de composición de mezclas minerales que permitan suministrar información para mejorar el proceso de sinterización.

\section{FABRICACIÓN DE SINTERIZADOS EN PLANTA PILOTO}

La empresa siderúrgica Arcelor_Mittal tiene instaladas en Gijón, Asturias, dos plantas de sinterización de 200 $\mathrm{m}^{2}$ y $281 \mathrm{~m}^{2}$ de superficie efectiva de sinterización, que producen anualmente 5,0-5,5 millones de toneladas de sínter. Este sínter se utiliza como material de carga en los dos hornos altos, instalados en Gijón, de $11,3 \mathrm{~m}$ de diámetro de crisol y $2.349 \mathrm{~m}^{3}$ de volumen de trabajo cada horno, que producen anualmente 3,8-4,3 millones de toneladas de arrabio.

En el presente trabajo de colaboración Arcelor_Mittal-CENIM, se han fabricado una serie de sinterizados en una planta piloto que el CENIM tiene instalada en Arcelor_Mittal en Gijón. La planta piloto tiene una paila de $40 \times 40 \mathrm{~cm}$ de sección transversal y $60 \mathrm{~cm}$ de altura y está equipada con un circuito de succión capaz de crear depresiones superiores a $1.470 \mathrm{mPa}$, un sistema de ignición de propano y un sistema para la medida del volumen de gas de entrada y la temperatura del gas de salida (Fig. 1)

\subsection{Materias primas utilizadas}

En el CENIM se han utilizado una serie de minerales de hierro, fundentes y coque que habitualmente se consumen en las plantas de sinterización de Arcelor_Mittal en Gijón.

La tabla I ofrece el análisis químico de los minerales de hierro. Todos presentan un contenido elevado en hierro, desde el Mount Wright y los concentrados MBR, con 66-67 \% de hierro, hasta el mineral español Andaluza con 53,7\% de hierro. Todos los minerales de hierro son de naturaleza hematítica.

Se utilizan caliza y dunita como fundentes:

- Caliza: 0,13\% Fe total, 55,44 \% CaO, 0,51\% $\mathrm{MgO}, 0,19 \% \mathrm{Al}_{2} \mathrm{O}_{3}, 0,42 \% \mathrm{SiO}_{2}$ y $44,97 \%$ de pérdidas por calcinación.

- Dunita: 5,83 \% Fe total, 2,32 \% CaO, 35,51 \% $\mathrm{MgO}, 2,82 \% \mathrm{Al}_{2} \mathrm{O}_{3}, 39,92 \% \mathrm{SiO}_{2}, 0,12 \%$ $\mathrm{MnO}$ y $8,27 \%$ de pérdidas por calcinación.

El coque se analizó en el Instituto Nacional del Carbón de Oviedo (INCAR) con el resultado siguiente: $83,50 \%$ carbono, $12,00 \%$ cenizas, $2,20 \%$ materia volátil, 0,95\% humedad, 0,25\% hidrógeno, 0,96 \% nitrógeno, 0,10 \% azufre y un índice de reactividad CRI de 60,46.

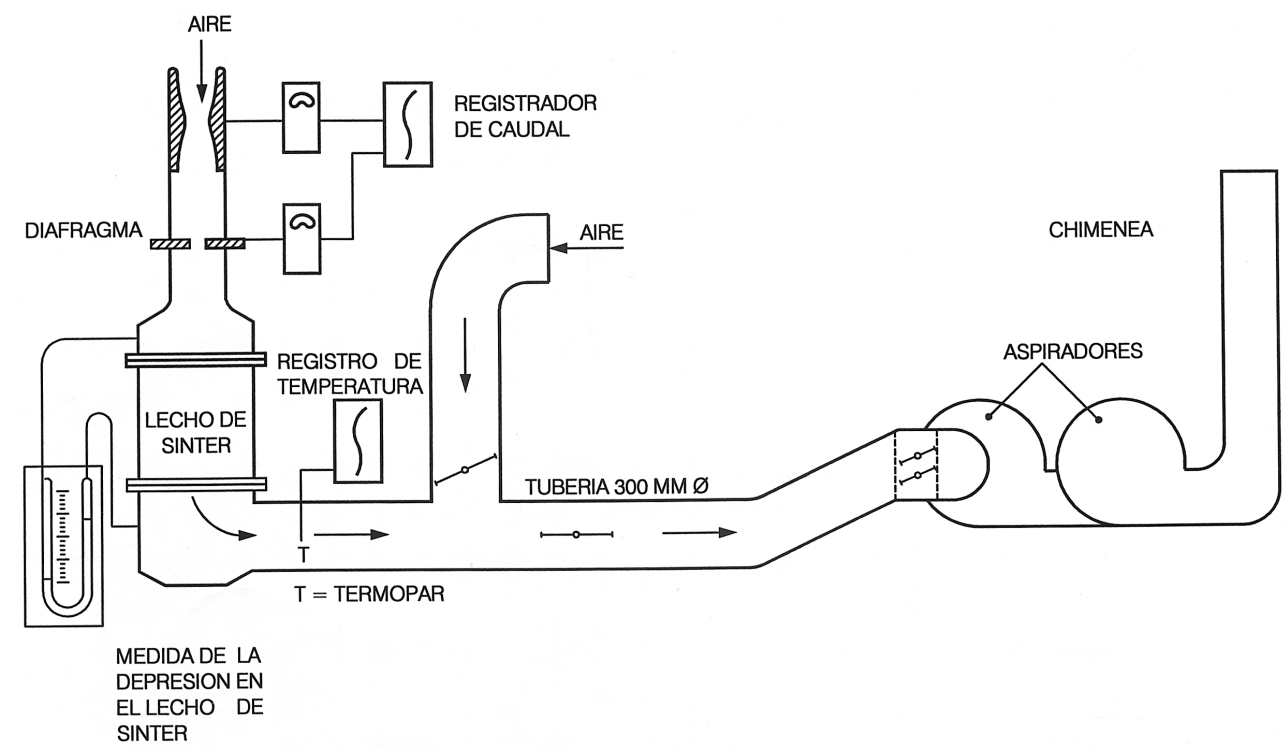

Figura 1. Planta piloto de sinterización.

Figure 1. Sintering pilot plant. 
RACIONALIZACIÓN DE MEZCLAS DE MINERALES DE HIERRO PARA LA OBTENCIÓN DE SINTERIZADOS DE CALIDAD OPTIMA RATIONALISATION OF IRON ORE MIXTURES FOR OBTAINING OPTIMUM QUALITY SINTERS

Tabla I. Análisis químico de minerales de hierro, \% en masa

Table I. Chemical analysis of iron ores, \% mass

\begin{tabular}{lccccccccccc}
\hline & Andaluza & CVRD- & CVRD- \\
Carajas & Tubarao & $\begin{array}{c}\text { San } \\
\text { Isidro }\end{array}$ & $\begin{array}{c}\text { Mount } \\
\text { Wright }\end{array}$ & $\begin{array}{c}\text { SNIM-T } \\
\text { Tazadit }\end{array}$ & $\begin{array}{c}\text { SNIM } \\
\text { Normal }\end{array}$ & $\begin{array}{c}\text { Goa } \\
\text { Silicioso }\end{array}$ & Nimco & Hamersly & $\begin{array}{c}\text { MBR } \\
\text { Brasil }\end{array}$ \\
\hline Fe total & 53,71 & 65,54 & 61,21 & 65,50 & 66,25 & 62,98 & 61,24 & 56,32 & 62,57 & 62,17 & 67,33 \\
$\mathrm{Fe}++$ & 0,0 & 0,27 & 0,55 & 0,61 & 1,61 & 0,40 & 0,86 & 4,83 & 3,04 & 0,27 & 0,69 \\
$\mathrm{CaO}$ & 4,12 & 0,10 & 0,04 & 0,04 & 0,13 & 0,11 & 0,12 & 0,07 & 0,03 & 0,10 & 0,07 \\
$\mathrm{MgO}$ & 0,64 & 0,03 & 0,02 & 0,03 & 0,08 & 0,02 & 0,08 & 0,09 & 0,02 & 0,06 & 0,04 \\
$\mathrm{Al}_{2} \mathrm{O}_{3}$ & 0,90 & 0,90 & 0,89 & 1,03 & 0,41 & 1,13 & 1,34 & 2,37 & 0,81 & 2,73 & 0,72 \\
$\mathrm{SiO}_{2}$ & 4,64 & 0,50 & 10,01 & 1,35 & 1,44 & 8,55 & 8,82 & 13,43 & 6,30 & 4,20 & 0,79 \\
$\mathrm{MnO}_{\mathrm{Na}} \mathrm{O}$ & 2,11 & 0,52 & 0,10 & 0,05 & 0,04 & 0,02 & 0,02 & 1,06 & 0,12 & 0,07 & 0,28 \\
$\mathrm{~K}_{2} \mathrm{O}$ & 0,013 & 0,011 & 0,006 & 0,005 & 0,011 & 0,017 & 0,024 & 0,026 & 0,013 & 0,034 & 0,007 \\
$\mathrm{~Pb}$ & 0,300 & 0,007 & 0,012 & 0,011 & 0,015 & 0,023 & 0,032 & 0,027 & 0,010 & 0,016 & 0,010 \\
$\mathrm{Zn}$ & 0,006 & 0,014 & 0,015 & 0,006 & 0,005 & 0,007 & 0,008 & 0,055 & 0,012 & 0,013 & 0,016 \\
$\mathrm{~S}$ & 0,005 & 0,007 & 0,011 & 0,004 & 0,003 & 0,004 & 0,009 & 0,010 & 0,004 & 0,005 & 0,011 \\
$\mathrm{P}_{2} \mathrm{O}_{5}$ & 0,012 & 0,011 & 0,005 & 0,025 & 0,005 & 0,004 & 0,004 & 0,007 & 0,006 & 0,016 & 0,005 \\
$\mathrm{P.C}$. & 0,057 & 0,160 & 0,087 & 0,069 & 0,057 & 0,057 & 0,057 & 0,064 & 0,149 & 0,137 & 0,085 \\
\hline
\end{tabular}

P.C. $=$ Pérdidas por calcinación .

\subsection{Composición de las mezclas minerales}

La tabla II ofrece el porcentaje en masa de los componentes de las mezclas minerales usadas para fabricar sinterizados. En todas las mezclas se utiliza el mineral Andaluza (a pesar de su menor ley en hierro), por ser el único mineral de la mezcla de origen español.

\subsubsection{Análisis químico}

La tabla III muestra el resultado del análisis químico de las mezclas minerales. El contenido en hierro es elevado para las mezclas 1 y 2 , como corresponde a los minerales presentes. El contenido en hierro de las mezclas 3 y 4 es tres unidades porcentuales menores, como consecuencia de introducir en las mezclas el mineral Goa Silicioso de menor contenido en hierro total. Los contenidos en álcalis y en ferroso son bajos.

\subsubsection{Análisis granulométrico}

El análisis granulométrico se ha realizado en un equipo ROT-TAP, en el que se produce el tamizado por desplazamiento de rotación excéntrica de vaivén, combinado con el sistema de percusión sobre la columna de tamices.
Tabla II. Composición de las mezclas minerales, \% en masa

Table II. Composition of ore mixtures, \% mass

\begin{tabular}{lrccc}
\hline Mezclas & $\mathbf{1}$ & $\mathbf{2}$ & $\mathbf{3}$ & $\mathbf{4}$ \\
\hline Andaluza & 4,0 & 10,3 & 10,3 & 10,3 \\
CVRD-Carajas & 25,0 & 27,8 & 21,3 & 24,4 \\
CVRD-Tubarao & - & $\overline{7}$ & 7,2 & 6,4 \\
San Isidro & 20,0 & 7,2 & $\overline{-}$ & - \\
Mount Wright & 8,0 & - & 7,2 & 7,2 \\
SNIM-Tazadit & 8,5 & 10,3 & - & - \\
SNIM-Normal & 4,0 & 6,2 & - & - \\
Goa Silicioso & 6,5 & - & 10,3 & 10,3 \\
Nimco & 4,5 & $\overline{12}$ & - & - \\
Hamersley & 4,0 & 12,4 & - & 10,3 \\
MBR Brasil & - & 10,3 & 20,6 & 10,3 \\
Caliza & - & - & 7,9 & 6,1 \\
Lodos LD & 2,6 & 2,6 & 2,1 & 2,1 \\
Escoria LD & 3,0 & 3,0 & 2,1 & 2,1 \\
Chatarra de hierro & 1,3 & 1,3 & 2,1 & 2,1 \\
Polvo del tragante & 0,7 & 0,7 & 0,6 & 0,6 \\
Finos de sínter de & & & & \\
retorno & 7,9 & 7,9 & 8,1 & 8,1 \\
\hline
\end{tabular}

En la figura 2 se ofrece la curva granulométrica para la mezcla mineral 1 , con un tamaño medio de partícula de 1,69 $\mathrm{mm}$. En la tabla IV se recogen estos valores para las cuatro mezclas. 
Tabla III. Análisis químico de las mezclas minerales, \% en masa

Table III. Chemical analysis of ore mixtures, \% mass

\begin{tabular}{lrrrr}
\hline & $\mathbf{1}$ & $\mathbf{2}$ & \multicolumn{1}{c}{$\mathbf{3}$} & \multicolumn{1}{c}{$\mathbf{4}$} \\
\hline $\mathrm{Fe}$ total & 58,64 & 62,28 & 56,01 & 55,62 \\
$\mathrm{Fe}++$ & 4,77 & 3,59 & 3,66 & 3,06 \\
$\mathrm{CaO}$ & 3,98 & 4,57 & 6,58 & 6,64 \\
$\mathrm{MgO}$ & 0,47 & 0,44 & 0,43 & 0,38 \\
$\mathrm{Al}_{2} \mathrm{O}_{3}$ & 1,20 & 1,04 & 0,90 & 1,18 \\
$\mathrm{SiO}_{2}$ & 4,26 & 3,46 & 4,22 & 4,38 \\
$\mathrm{MnO}$ & 0,57 & 0,69 & 0,80 & 0,77 \\
$\mathrm{Na}_{2} \mathrm{O}$ & 0,016 & 0,015 & 0,028 & 0,021 \\
$\mathrm{~K}_{2} \mathrm{O}$ & 0,044 & 0,054 & 0,038 & 0,058 \\
$\mathrm{~Pb}$ & 0,018 & 0,015 & 0,008 & 0,005 \\
$\mathrm{Zn}$ & 0,049 & 0,044 & 0,036 & 0,036 \\
$\mathrm{~S}$ & 0,026 & 0,023 & 0,030 & 0,028 \\
$\mathrm{P}_{2} \mathrm{O}_{5}$ & 0,073 & 0,082 & 0,071 & 0,121 \\
$\mathrm{P} . \mathrm{C}$. & 5,49 & 4,41 & 7,63 & 7,91 \\
\hline
\end{tabular}

P.C. = Pérdidas por calcinación.

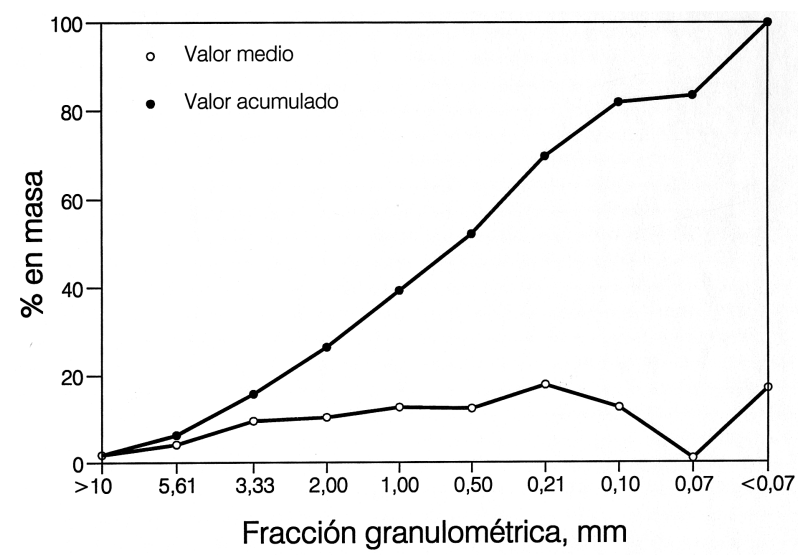

Figura 2. Análisis granulométrico de la mezcla mineral 1.

Figure 2. Granulometric analysis of ore mixture

\subsubsection{Aptitud a la granulación}

El tratamiento para la granulación es de importancia básica en la sinterización de minerales de hierro, puesto que una buena permeabilidad del lecho de sinterización determina, en una gran extensión, la velocidad a la cual el proceso progresa y con ello la productividad de la planta.

El ensayo de la aptitud a la granulación de cada mineral de hierro y de cada mezcla mineral, se ha
Tabla IV. Tamaño medio de partícula, reductibilidad y temperaturas de reblandecimiento y fusión de mezclas minerales

Table IV. Particle average size, reductibility and softening and melting temperatures of ore mixtures

\begin{tabular}{|c|c|c|c|c|}
\hline & 1 & 2 & 3 & 4 \\
\hline $\begin{array}{l}\text { Tamaño medio de } \\
\text { partícula, mm } \\
\text { Tamaño medio } \\
\text { después del ensayo }\end{array}$ & 1,69 & 1,90 & 1,66 & 1,78 \\
\hline SAFE, mm & 3,26 & 3,39 & 3,18 & 3,20 \\
\hline $\begin{array}{l}\text { Reductibilidad, } \mathrm{R}_{60}, \% \\
\text { Temp. de }\end{array}$ & 53,0 & 53,4 & 50,3 & 56,3 \\
\hline $\begin{array}{l}\text { reblandecimiento, } \\
\text { ST, }{ }^{\circ} \mathrm{C} \\
\text { Temp. de fusion, }\end{array}$ & 1.607 & 1.599 & - & - \\
\hline $\mathrm{FT},{ }^{\circ} \mathrm{C}$ & 1.635 & 1.620 & - & - \\
\hline
\end{tabular}

realizado siguiendo el procedimiento estándar SAFE (Size Analysis on Frozen Elements) establecido por el Centro Sviluppo Materiali (CSM) de Italia ${ }^{[4]}$.

La mezcla mineral se somete al tratamiento de granulación antes de la sinterización para aumentar el tamaño medio de los minerales que tienen una granulometría muy fina, intentando mejorar el comportamiento dinámico durante la sinterización.

En la figura 3 se representa la curva granulométrica de la mezcla mineral 1 inicial y después del ensayo SAFE.

En la tabla IV se ofrece el valor medio del tamaño de las partículas de las mezclas minerales después

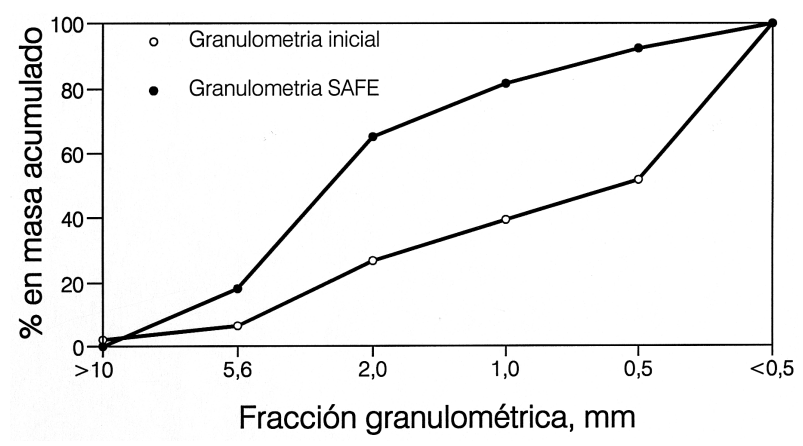

Figura 3. Aptitud a la granulación de la mezcla mineral 1.

Figure 3. Granulation fitness of ore mixture 1. 
de haber sido sometidas al ensayo SAFE. Todas las mezclas presentan una buena aptitud a la granulación.

\subsubsection{Reductibilidad}

El ensayo de reductibilidad se ha realizado a $900^{\circ} \mathrm{C}$ siguiendo el estándar ISO para sinterizados, adaptado para las mezclas minerales de la fracción de 3,3-2,0 $\mathrm{mm}{ }^{[5]}$. Los resultados obtenidos se recogen en la tabla IV. El valor de $\mathrm{R}_{60}$ oscila entre 50 y $56 \%$ y se puede considerar como bueno.

En la figura 4 se muestra la curva que se obtiene al someter la mezcla mineral 1 al ensayo de reductibilidad.

\subsubsection{Temperaturas de reblandecimiento y fusión}

El ensayo para determinar las temperaturas de reblandecimiento y fusión de las mezclas minerales se ha realizado utilizando un equipo LECO AF-600, con un horno que alcanza una temperatura máxima de $1.650^{\circ} \mathrm{C}$, en el cual se han fijado los parámetros siguientes con la ayuda de un ordenador: estándar ASTM con atmósfera inerte $\left(\mathrm{N}_{2}\right)$, programa de inicio y fin de las temperaturas, flujo de gas y velocidad de calentamiento ${ }^{[6]}$.

La tabla IV indica los puntos representativos para las mezclas 1 y 2 . Las temperaturas de fusión son elevadas. La diferencia entre la temperatura de reblandecimiento y fusión es pequeña y, ello, es beneficioso para el comportamiento del sinterizado en la zona cohesiva del horno alto.

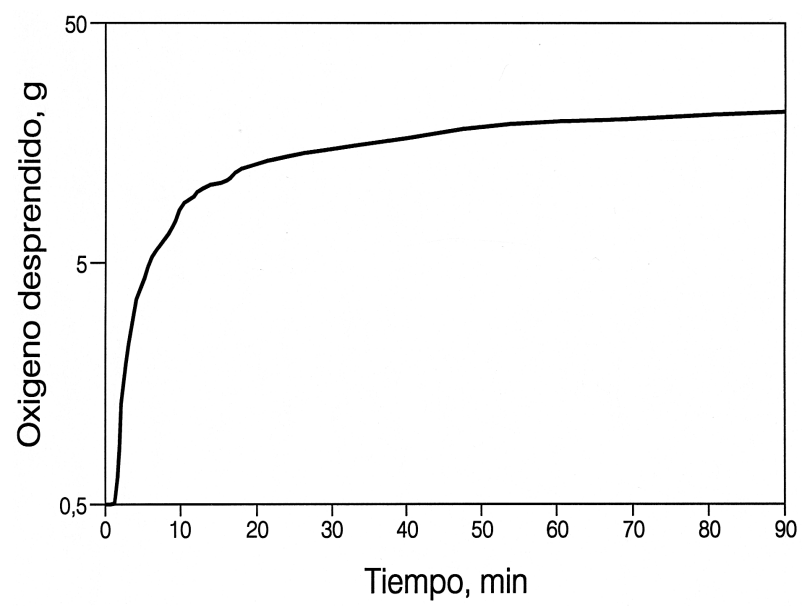

Figura 4. Reductibilidad de la mezcla mineral 1.

Figure 4. Reductibility of ore mixture 1.

\subsubsection{Termoanálisis}

El comportamiento térmico de las mezclas minerales se ha estudiado en una termobalanza SETARAM, que proporciona un registro gráfico de las variaciones en la temperatura, peso, velocidad de variación de peso y entalpía (análisis termogravimétrico TG, diferencial del análisis termogravimétrico DTG y análisis térmico diferencial ATD).

Las figuras 5 a) y 5 b) muestran el termoanálisis de la mezcla mineral 1 , realizado en una atmósfera inerte $\left(\mathrm{N}_{2}\right)$. El efecto endotérmico registrado entre 250 y $400^{\circ} \mathrm{C}$, con un pico a $320^{\circ} \mathrm{C}$, conduce a una pérdida de masa importante, debida al desprendimiento de agua de constitución de la goetita, según la reacción:

$$
\mathrm{Fe}_{2} \mathrm{O}_{3} \cdot \mathrm{H}_{2} \mathrm{O} \rightarrow \mathrm{Fe}_{2} \mathrm{O}_{3}+\mathrm{H}_{2} \mathrm{O}
$$

que viene a representar un $1,8 \%$. El segundo efecto endotérmico importante es el registrado entre 600 y $860^{\circ} \mathrm{C}$, con un pico a $745-750^{\circ} \mathrm{C}$, con una pérdida de masa del 2,3 \% (Fig. 5b)). Este efecto y la pérdida de masa correspondiente representan la pérdida de
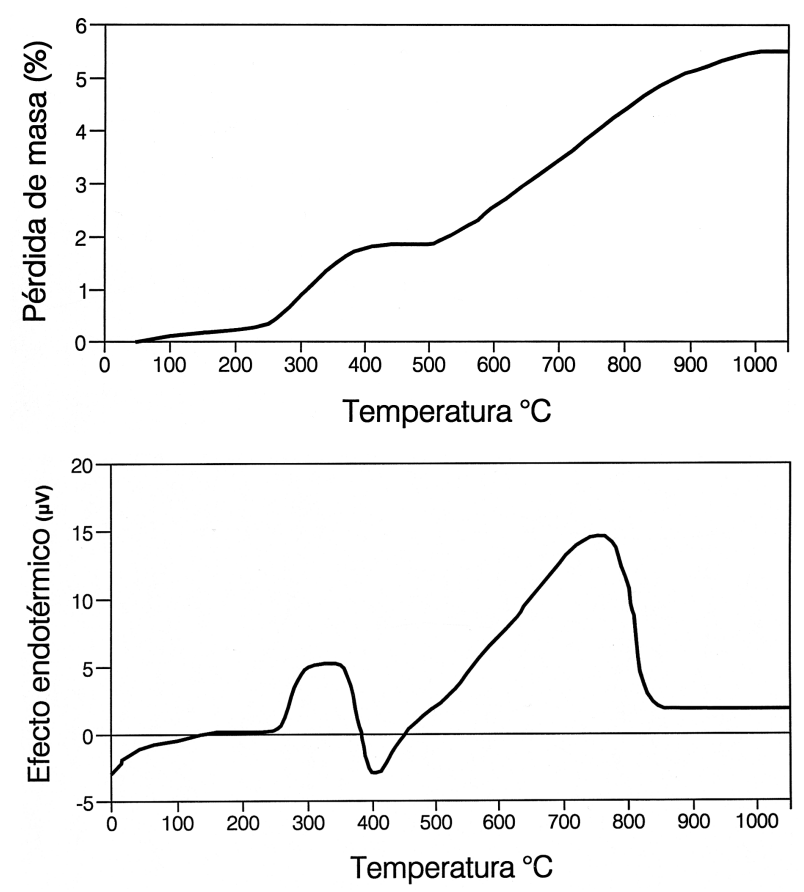

Figura 5. a) Análisis termogravimétrico de la mezcla mineral 1 ; b) Análisis térmico diferencial de la mezcla mineral 1.

Figure 5 a) Thermogravimetric analisys of ore mixture 1 ; b) Differential thermal analysis of ore mixture 1. 
$\mathrm{CO}_{2}$ producida por la descomposición de calcita, magnesita y dolomita presentes en este tipo de muestras de mineral de hierro.

La pequeña pérdida de masa registrada en la curva TG entre 400 y $600{ }^{\circ} \mathrm{C}$ y entre 800 y $1.000^{\circ} \mathrm{C}$, sin que tenga un efecto preciso sobre la curva ATD, puede ser debida al desprendimiento de agua de constitución de algún tipo de silico-aluminato hidratado presente en la mezcla mineral (Fig. 5 a)). Estos silicoaluminatos pueden ser: caolinita, gibsita, pirofilita y ferrihaloisita. Debido a la pequeña cantidad presente de estos compuestos, pueden considerarse como elementos minoritarios y que junto con la sílice forman la ganga del mineral.
Las mezclas minerales 2,3 y 4 presentan un comportamiento térmico similar a la mezcla 1 .

\subsection{Fabricación de sinterizados}

Se han fabricado una serie de sinterizados en la planta piloto (Fig. 1) usando las mezclas minerales (Tabla III). En el proceso de fabricación se han mantenido constantes varios de los parámetros de operación, de acuerdo con la práctica industrial, tales como:

(i) depresión del trabajo : $1.470 \mathrm{mPa}$

(ii) aporte calorífico durante la ignición: $12.480 \mathrm{~kJ}$

Tabla V. Sintetizados fabricados en planta piloto con la mezcla mineral 1

Table V. Sinterings made in pilot plant with the ore mixture 1

\begin{tabular}{|c|c|c|c|c|c|c|c|c|c|}
\hline \multicolumn{2}{|c|}{ Sinterizado, núm. } & $\mathrm{S} 2$ & S4 & S6 & S8 & S10 & $\mathrm{S} 12$ & S14 & S16 \\
\hline \multicolumn{2}{|l|}{ Altura de lecho, $\mathrm{cm}$} & 50 & 50 & 50 & 50 & 60 & 60 & 60 & 60 \\
\hline \multicolumn{2}{|l|}{ Finos de retorno, \% } & 25 & 25 & 35 & 35 & 25 & 25 & 35 & 35 \\
\hline \multicolumn{2}{|l|}{ Basicidad, $\mathrm{CaO} / \mathrm{SiO}_{2}$} & 1,6 & 1,9 & 1,6 & 1,9 & 1,6 & 1,9 & 1,6 & 1,9 \\
\hline \multicolumn{2}{|l|}{ Mezcla Mineral 1, kg } & 124,0 & 113,6 & 113,5 & 113,5 & 139,4 & 134,3 & 134,3 & 129,1 \\
\hline \multicolumn{2}{|l|}{ Dunita, kg } & 2,0 & 1,9 & 1,7 & 1,6 & 2,6 & 2,6 & 2,3 & 2,0 \\
\hline \multicolumn{2}{|l|}{ Coque, $\mathrm{kg}$} & 5,4 & 4,7 & 4,2 & 4,4 & 5,9 & 5,7 & 5,3 & 5,1 \\
\hline \multicolumn{2}{|l|}{ Finos de retorno, kg } & 30,0 & 27,5 & 38,5 & 38,5 & 33,8 & 32,5 & 45,5 & 43,8 \\
\hline \multicolumn{2}{|l|}{ Caliza, kg } & 9,6 & 13,2 & 9,9 & 13,0 & 12 & 16,1 & 11,9 & 16,4 \\
\hline \multicolumn{2}{|l|}{ Agua, kg } & 9,5 & 8,8 & 8,8 & 8,8 & 10,0 & 9,4 & 10,0 & 10,5 \\
\hline \multicolumn{2}{|l|}{ Total peso mezcla, kg } & 180,5 & 169,7 & 176,6 & 179,8 & 204,0 & 200,6 & 209,3 & 206,9 \\
\hline \multirow{5}{*}{ Granulometria, mm } & $>40$ & 48,9 & 35,8 & $36, \varsigma$ & 35 & 61,2 & 54 & 56,7 & 47,8 \\
\hline & $40-20$ & g 17,2 & 29,6 & 21,6 & 23,4 & 26,2 & 3 & 23,0 & 27,3 \\
\hline & $20-12, \mathrm{~kg}$ & 8,8 & 12,4 & 8,3 & 9,3 & 12,0 & 12,3 & 8,2 & 11,6 \\
\hline & $12-5, \mathrm{~kg}$ & g 31,3 & 28,1 & 33,3 & 31,0 & 32,5 & 28,0 & 33,6 & 36,6 \\
\hline & & 28,6 & 26,1 & 36,2 & 34,4 & 32,6 & 31,0 & 42,0 & 40,0 \\
\hline \multicolumn{2}{|l|}{ Total peso torta, kg } & 1 & & & & & & & \\
\hline \multicolumn{2}{|l|}{ Sinter útil, kg } & 10 & & 10 & & & & & 123,3 \\
\hline \multicolumn{2}{|l|}{ Finos de retorno, $\mathrm{kg}$} & 28 & 26 & 36 & 34 & 32 & & 42,0 & 40,0 \\
\hline \multicolumn{2}{|l|}{ Tamaño medio, mm } & 33,7 & 31,1 & 29,1 & 28,9 & 42,9 & 41,9 & 39,4 & 37,0 \\
\hline \multirow{3}{*}{\multicolumn{2}{|c|}{$\begin{array}{l}\text { Densidad de carga, } \mathrm{kg} / \mathrm{m}^{3} \\
\text { Depresión inicial, mPascal } \\
\text { Depresión total, mPascal }\end{array}$}} & .997 & .984 & 1.987 & .967 & 1.977 & 1.9651 & 1.965 & 1.981 \\
\hline & & 804 & 764 & 794 & 794 & 960 & 960 & 931 & 882 \\
\hline & & 1.470 & $\begin{array}{ll}1470 & 1\end{array}$ & 1.470 & $1.470 \quad 1$ & $1.470 \quad 1$ & $1.470 \quad 1$ & 1.470 & 1.470 \\
\hline \multicolumn{2}{|c|}{ Temp. máxima humos, ${ }^{\circ} \mathrm{C}$} & 305 & 380 & 340 & 360 & 350 & 360 & 370 & 380 \\
\hline \multicolumn{2}{|l|}{ Permeabilidad,minutos } & 1,73 & 1,87 & 1,87 & 1,87 & 1,88 & 1,88 & 1,87 & $1, \varepsilon$ \\
\hline \multicolumn{2}{|c|}{ Consumo coque, $\mathrm{kg} / \mathrm{t}$ de sínter } & 45,1 & 41,7 & 37,9 & 38,9 & 42,8 & 42,2 & 40,0 & 38,7 \\
\hline \multicolumn{2}{|c|}{ Productividad, $\mathrm{t} / \mathrm{m}^{2} .24 \mathrm{~h}$} & 40,1 & 38,3 & 36,2 & 37,5 & 36,0 & 36,6 & 33,5 & 37,1 \\
\hline
\end{tabular}


(iii) tiempo de ignición : $90 \mathrm{~s}$

(iv) altura de sobreparrilla $: 3 \mathrm{~cm}$

mientras que otros parámetros han sido variables:

(i) altura del lecho: 50 y $60 \mathrm{~cm}$

(ii) finos de retorno : 25 y $35 \%$

(iii) índice de basicidad $\left(\mathrm{CaO} / \mathrm{SiO}_{2}\right): 1,6$ y 1,9

\section{RESULTADOS}

Las tablas V y VI muestran los resultados de los sinterizados fabricados con las mezclas minerales 1 y 2 , respectivamente.
Los mejores sinterizados desde el punto de vista de la resistencia fueron conseguidos tanto para la mezcla 1 como para la mezcla 2 , con unas condiciones de marcha de $60 \mathrm{~cm}$ de altura de capa, $25 \%$ de finos de retorno y 1,9 de basicidad. El índice Tumbler fue superior a 72 en los sinterizados producidos con las condiciones anteriores de marcha. Se supone que cuando la basicidad aumenta de 1,6 a 1,9 se obtiene una estructura de sinterizado con una textura mas heterogénea, lo que le confiere una resistencia mas elevada. Con estas condiciones de marcha se obtiene un grado de reducción $\mathrm{R}_{60}$ bueno y un índice $\mathrm{RDI}$ malo para la mezcla 1 . Para la mezcla 2 los valores de $\mathrm{R}_{60}$ y RDI son buenos.

Tabla VI. Sintetizados fabricados en planta piloto con la mezcla mineral 2

Table VI. Sinterings made in pilot plant with the ore mixture 2

\begin{tabular}{|c|c|c|c|c|c|c|c|c|c|}
\hline \multicolumn{2}{|c|}{ Sinterizado, núm. } & S18 & S20 & S22 & S24 & \multicolumn{2}{|r|}{ S28 } & S30 & S32 \\
\hline \multicolumn{2}{|l|}{ Altura de lecho, $\mathrm{cm}$} & 50 & 50 & 50 & 50 & 60 & 60 & 60 & 60 \\
\hline \multicolumn{2}{|l|}{ Finos de retorno, \% } & 25 & 25 & 35 & 35 & 25 & 25 & 35 & 35 \\
\hline \multicolumn{2}{|l|}{ Basicidad, $\mathrm{CaO} / \mathrm{SiO}_{2}$} & 1,6 & 1,9 & 1,6 & 1,9 & 1,6 & 1,9 & 1,6 & 1,9 \\
\hline \multicolumn{2}{|l|}{ Mezcla Mineral 2, kg } & 112,3 & 112,3 & 112,3 & 112,3 & 138,1 & 138,1 & 138,1 & 138,1 \\
\hline \multicolumn{2}{|l|}{ Dunita, kg } & 2,2 & 2,0 & 2,0 & 1,9 & 2,8 & 2,7 & 2,3 & 2,3 \\
\hline \multicolumn{2}{|l|}{ Coque, kg } & 5,0 & 4,8 & 4,4 & 4,5 & 5,8 & 5,9 & 5,5 & 5,8 \\
\hline \multicolumn{2}{|l|}{ Finos de retorno, $\mathrm{kg}$} & 27,5 & 27,5 & 38,5 & 38,5 & 33,8 & 33,8 & 47,3 & 47,3 \\
\hline \multicolumn{2}{|l|}{ Caliza, kg } & 10,1 & 13,1 & 8,9 & 12,9 & 11,9 & 16 & 10,8 & 16,5 \\
\hline \multicolumn{2}{|l|}{ Agua, kg } & 8,5 & 8,7 & 9,0 & 9,5 & 11,0 & 11,0 & 11,5 & 11,8 \\
\hline \multicolumn{2}{|l|}{ Total peso mezcla, kg } & 165,6 & 168,4 & 175,1 & 179,6 & 203,4 & 207,5 & 215,5 & 221,8 \\
\hline & $>40, \mathrm{~kg}$ & 43,7 & 38,0 & 38,9 & 36,7 & 57,6 & 42,0 & 55,1 & 51,1 \\
\hline & $40-20, \mathrm{~kg}$ & 27,3 & 33,2 & 20,8 & 24,8 & 27,4 & 37,8 & 23,3 & 30,6 \\
\hline \multirow[t]{3}{*}{ Granulometria, mm } & $20-12, \mathrm{~kg}$ & 9,9 & 11,2 & 7,8 & 9,2 & 11,5 & 14,5 & 8,5 & 10,0 \\
\hline & $12-5, \mathrm{~kg}$ & g 25,8 & 24,3 & 31,9 & 29,2 & 34,6 & 33,7 & 34,4 & 33,6 \\
\hline & $<5, \mathrm{~kg}$ & 25,2 & 24,8 & 37,2 & 33,7 & 33,8 & 32,6 & 46,5 & 37,0 \\
\hline \multicolumn{2}{|l|}{ Total peso torta, kg } & 131,9 & 131,4 & 136,6 & 133,5 & 164,8 & 160,6 & 167,7 & 162,3 \\
\hline \multicolumn{2}{|l|}{ Sínter útil, kg } & 106,7 & 106,6 & 99,4 & 99,8 & 131,0 & 128,0 & 121,3 & 125,3 \\
\hline \multicolumn{2}{|l|}{ Finos de retorno, kg } & 25,2 & 24,8 & 37,2 & 33,7 & 33,8 & 32,6 & 46,5 & 37,0 \\
\hline \multicolumn{2}{|l|}{ Tamaño medio, mm } & 33,8 & 32,8 & 29,6 & 29,7 & 41,8 & 37,5 & 38,8 & 39,2 \\
\hline \multirow{3}{*}{\multicolumn{2}{|c|}{$\begin{array}{l}\text { Densidad de carga, } \mathrm{kg} / \mathrm{m}^{3} \\
\text { Depresión inicial, mPascal } \\
\text { Depresión total, mPascal }\end{array}$}} & 1.949 & 1.957 & 1.983 & 1.957 & 2.011 & 1.971 & $1.996 \quad 1$ & 1.964 \\
\hline & & 804 & 862 & 784 & 784 & 862 & 862 & 921 & 902 \\
\hline & & 1.470 & 1.4701. & 1.470 & 1.470 & 1.470 & 1.470 & 1.470 & 1.470 \\
\hline \multicolumn{2}{|c|}{ Temp. máxima humos, ${ }^{\circ} \mathrm{C}$} & 350 & 370 & 380 & 380 & 340 & 380 & 400 & 400 \\
\hline \multicolumn{2}{|c|}{ Permeabilidad, minutos } & 2,01 & 2,04 & 2,02 & 2,02 & 2,02 & 1,57 & 1,55 & 1,53 \\
\hline \multicolumn{2}{|c|}{ Consumocoque,kg/tde sínter } & 44,2 & 42,0 & 40,2 & 39,4 & 42,8 & 42,8 & 41,0 & 40,0 \\
\hline \multicolumn{2}{|c|}{ Productividad, $\mathrm{t} / \mathrm{m}^{2} .24 \mathrm{~h}$} & 39,6 & 39,4 & 37,2 & 37,2 & 40,5 & 41,8 & 34,9 & 39,4 \\
\hline
\end{tabular}


Al comparar los resultados obtenidos con la mezcla mineral 1 y con la mezcla mineral 2 , fue posible establecer las variables que permiten fabricar los sinterizados de mejor calidad para las mezclas minerales 3 y 4 (Tabla VII). Los parámetros de operación para estas mezclas son:

(i) altura del lecho: $60 \mathrm{~cm}$

(ii) finos de retorno: $25 \%$

(iii) basicidad : 1,9

Las tablas V-VII muestran las variables de operación y las características de los sinterizados fabricados.

El peso total de la mezcla cargada en la paila aumenta con la altura del lecho de 170 a $210 \mathrm{~kg}$.

\subsection{Tamaño medio de los sinterizados}

Las tablas V-VII indican el peso de las diferentes fracciones granulométricas de cada sinterizado, cuyo tamaño medio varía de 30 a $42 \mathrm{~mm}$.

\subsection{Consumo de coque}

El consumo de coque es un parámetro a tener en cuenta por la repercusión en el precio del arrabio, ya que el número de toneladas que se mueven en la siderurgia hace que sea un factor muy importante en la rentabilidad de la misma.

Tabla VII. Sintetizados fabricados en planta piloto con las mezclas minerales 3 y 4

Table VII. Sinterings made in pilot plant with the ore mixtures 3 and 4

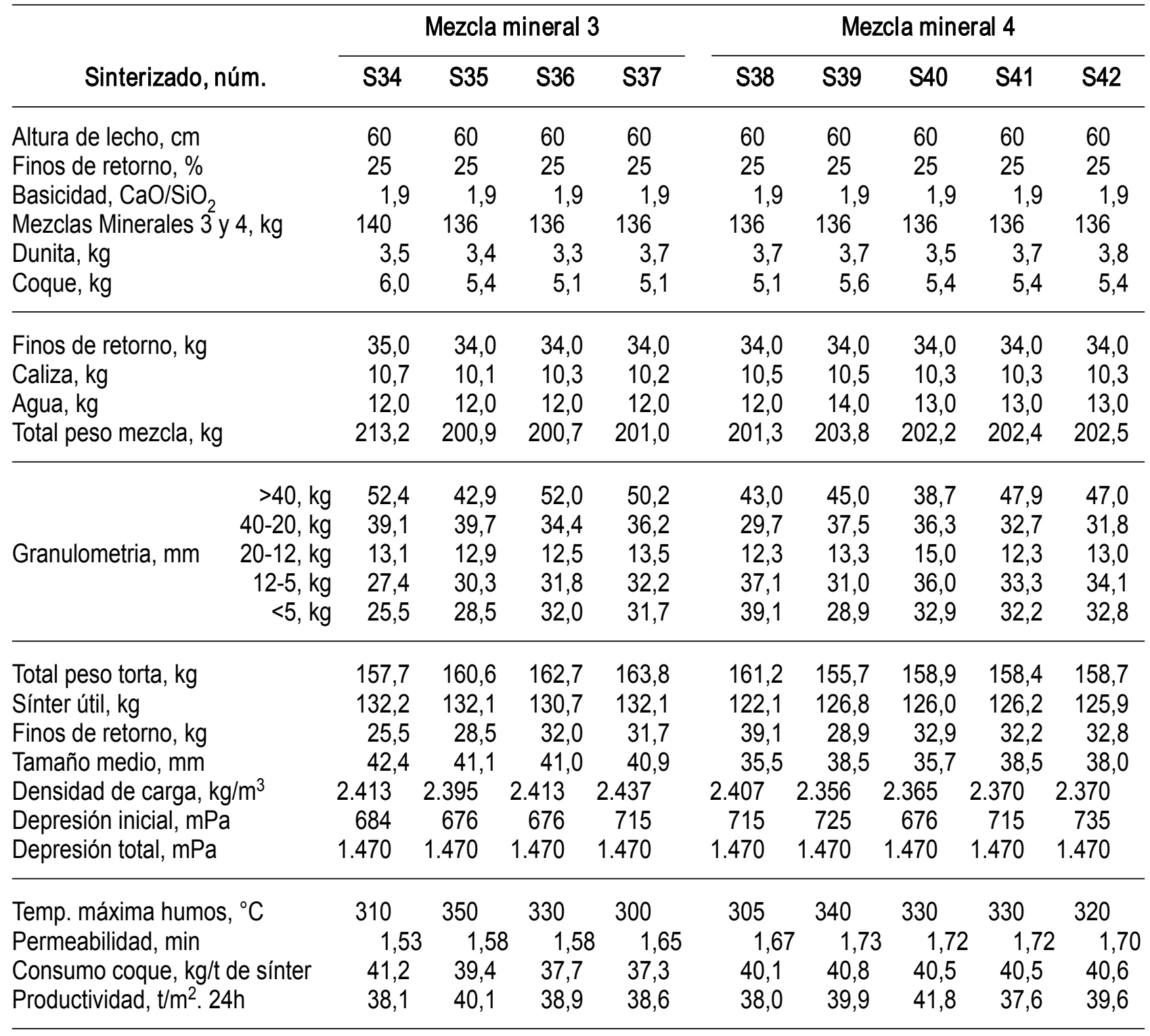


Para las mezclas minerales 1 y 2 (Tablas V y VI) se aprecia como el aumento de la basicidad hace que descienda el consumo de coque. Los consumos son más reducidos cuando se trabaja con retornos del 35 $\%$ en vez del $25 \%$, haciéndose más sensible esta disminución cuando se trabaja con una altura de capa de $50 \mathrm{~cm}$ que con una altura de $60 \mathrm{~cm}$.

En las tablas V-VII, se observa que el consumo de coque oscila entre 38 y $45 \mathrm{~kg} / \mathrm{t}$ sínter.

\subsection{Productividad}

La productividad es un objetivo primordial de las plantas de sinterización que tienen que abastecer las necesidades de los hornos altos, sin que el aumento de la productividad signifique una disminución de la calidad del sinterizado.

Para las mezclas 1 y 2 (Tablas V y VI) se aprecia un aumento de la productividad a medida que la basicidad se eleva de 1,6 a 1,9. También, se observa que esta influencia se hace más sensible con retornos del $35 \%$ y altura de capa de $60 \mathrm{~cm}$ que cuando se hace en las condiciones restantes de marcha.

En las tablas V-VII se observa que la productividad permanece entre 34 y $42 \mathrm{Mg} / \mathrm{m}^{2}$ día)

\subsection{Análisis químico de los sinterizados}

La tabla VIII ofrece el resultado del análisis químico de los sinterizados. El contenido en hierro total es elevado (50-58 \%) y el contenido en $\mathrm{FeO}$ es bajo

Tabla VIII. Análisis químico de los sinterizados, \% en masa

Table VIII. Chemical analysis of sinters, \% mass

\begin{tabular}{|c|c|c|c|c|c|c|c|c|c|c|c|c|}
\hline $\begin{array}{l}\text { Mezcla } \\
\text { Mineral }\end{array}$ & Sínter & Fe total & $\mathrm{FeO}$ & $\mathrm{SiO}_{2}$ & $\mathrm{Al}_{2} \mathrm{O}_{3}$ & $\mathrm{CaO}$ & $\mathrm{MgO}$ & $\mathrm{MnO}$ & $S$ & $P$ & $\mathrm{Na}_{2} \mathrm{O} \quad \mathrm{K}_{2} \mathrm{O}$ & $\begin{array}{l}\mathrm{CaOl} \\
\mathrm{SiO}_{2}\end{array}$ \\
\hline \multirow{8}{*}{1} & $\mathrm{~S} 2$ & 57,5 & 5,5 & 5,7 & 1,16 & 9,15 & 1,55 & 0,64 & 0,012 & 0,034 & $0,0410,066$ & 1,62 \\
\hline & S4 & 56,3 & 5,7 & 5,6 & 1,35 & 10,45 & 1,71 & 0,72 & 0,013 & 0,041 & $0,038 \quad 0,060$ & 1,87 \\
\hline & S6 & 57,1 & 3,5 & 5,6 & 1,17 & 9,25 & 1,63 & 0,66 & 0,010 & 0,030 & $0,0390,064$ & 1,59 \\
\hline & S8 & 56,0 & 3,2 & 5,4 & 1,15 & 11,10 & 1,53 & 0,64 & 0,017 & 0,031 & $0,038 \quad 0,065$ & 2,02 \\
\hline & S10 & 57,1 & 5,0 & 5,8 & 1,24 & 9,20 & 1,44 & 0,65 & 0,012 & 0,041 & $0,038 \quad 0,058$ & 1,59 \\
\hline & $\mathrm{S} 12$ & 56,2 & 4,6 & 5,7 & 1,15 & 10,90 & 1,58 & 0,68 & 0,015 & 0,039 & $0,0390,058$ & 1,93 \\
\hline & S14 & 57,5 & 3,3 & 5,4 & 1,20 & 9,10 & 1,51 & 0,66 & 0,011 & 0,038 & $0,037 \quad 0,060$ & 1,70 \\
\hline & S16 & 56,7 & 4,3 & 5,5 & 1,17 & 10,40 & 1,50 & 0,70 & 0,018 & 0,040 & $0,037 \quad 0,056$ & 1,90 \\
\hline \multirow{8}{*}{2} & S18 & 57,3 & 5,7 & 5,4 & 1,22 & 9,35 & 1,70 & 0,74 & 0,011 & 0,042 & $0,0390,071$ & 1,73 \\
\hline & S20 & 56,6 & 5,6 & 5,3 & 1,17 & 10,50 & 1,68 & 0,72 & 0,014 & 0,039 & $0,037 \quad 0,071$ & 1,98 \\
\hline & S22 & 57,6 & 3,7 & 5,4 & 1,19 & 8,80 & 1,60 & 0,80 & 0,013 & 0,040 & $0,036 \quad 0,072$ & 1,64 \\
\hline & S24 & 56,9 & 3,6 & 4,9 & 1,16 & 10,35 & 1,61 & 0,71 & 0,018 & 0,040 & $0,031 \quad 0,068$ & 2,13 \\
\hline & S26 & 58,0 & 4,7 & 5,2 & 1,20 & 8,60 & 1,62 & 0,75 & 0,012 & 0,040 & $0,032 \quad 0,066$ & 1,67 \\
\hline & S28 & 56,5 & 5,0 & 5,7 & 1,10 & 10,13 & 1,66 & 0,73 & 0,014 & 0,036 & $0,036 \quad 0,072$ & 1,82 \\
\hline & S30 & 56,7 & 3,5 & 5,0 & 1,19 & 9,05 & 1,56 & 0,79 & 0,012 & 0,041 & $0,040 \quad 0,075$ & 1,81 \\
\hline & S32 & 57,0 & 4,5 & 5,5 & 1,17 & 9,90 & 1,49 & 0,77 & 0,015 & 0,039 & $0,0390,076$ & 1,80 \\
\hline \multirow{4}{*}{3} & S34 & 55,5 & 5,2 & 5,5 & 0,99 & 10,85 & 1,65 & 0,96 & 0,022 & 0,030 & $0,042 \quad 0,070$ & 1,97 \\
\hline & S35 & 56,2 & 5,5 & 5,7 & 0,91 & 10,85 & 1,55 & 0,94 & 0,017 & 0,031 & $\begin{array}{lll}0,038 & 0,064\end{array}$ & 1,90 \\
\hline & S36 & 56,0 & 3,8 & 5,6 & 1,06 & 10,80 & 1,65 & 0,93 & 0,023 & 0,035 & $0,045 \quad 0,078$ & 1,93 \\
\hline & S37 & 55,4 & 3,9 & 5,8 & 1,11 & 11,10 & 1,68 & 0,99 & 0,020 & 0,029 & $0,042 \quad 0,078$ & 1,91 \\
\hline \multirow{5}{*}{4} & S38 & 54,9 & 3,5 & 6,0 & 1,15 & 11,55 & 1,68 & 0,93 & 0,025 & 0,032 & $0,041 \quad 0,079$ & 1,94 \\
\hline & S39 & 55,7 & 5,5 & 5,6 & 1,12 & 11,55 & 1,88 & 0,85 & 0,015 & 0,035 & $0,041 \quad 0,092$ & 2,01 \\
\hline & S40 & 56,1 & 5,3 & 5,3 & 1,18 & 10,10 & 1,70 & 0,80 & 0,012 & 0,034 & $0,0390,091$ & 1,91 \\
\hline & S41 & 56,5 & 5,2 & 5,2 & 1,22 & 10,10 & 1,69 & 0,97 & 0,014 & 0,032 & $0,042 \quad 0,088$ & 1,94 \\
\hline & S42 & 55,3 & 4,4 & 5,7 & 1,57 & 11,00 & 2,01 & 0,80 & 0,015 & 0,037 & $0,044 \quad 0,095$ & 1,95 \\
\hline
\end{tabular}


(3,2-5,7\%) que destaca la naturaleza hematítica del óxido de hierro. Los contenidos de los elementos perjudiciales tales como azufre, fósforo y álcalis son bajos, indicando que los sinterizados son adecuados para su utilización en el horno alto.

\subsection{Ensayo RDI}

La degradación del sinterizado durante la reducción a baja temperatura se determina por el ensayo RDI (reducción degradation index) [7]. El RDI de los sinterizados fabricados oscila entre 33 y 49 (Tabla IX). Es deseable valores bajos para este índice. La degradación del sinterizado tiene lugar durante la reducción en el horno alto, en la zona de baja temperatura (850. $950^{\circ} \mathrm{C}$ ) y causa un efecto perjudicial sobre la resistencia de la carga en el horno con la subsiguiente pérdida de permeabilidad de los gases reductores y aumento del consumo de coque ${ }^{[8]}$.

La degradación se origina en la transformación, hasta una cierta extensión, que tiene lugar durante

Tabla IX. Ensayos RDI, Tumbler, reductibilidad y de temperaturas de reblandecimiento (ST) y fusión (FT)

Table IX. RDI, Tumber, reductivility and softening (ST) and meeting (FT) temperatures tests.

\begin{tabular}{|c|c|c|c|c|c|c|c|c|}
\hline \multirow[t]{2}{*}{ Mezcla mineral } & \multirow[t]{2}{*}{ Sínter } & \multirow{2}{*}{$\begin{array}{c}\mathrm{RDI} \\
(<3 \mathrm{~mm}) \\
\%\end{array}$} & \multirow{2}{*}{$\begin{array}{c}\text { Tumbler } \\
\text { (>6.3mm), } \\
\%\end{array}$} & \multicolumn{2}{|c|}{ Reductibilidad } & \multicolumn{3}{|c|}{ Reblandecimiento y fusión } \\
\hline & & & & $\begin{array}{l}\text { Velocidad de } \\
\text { reducción } \\
(\mathrm{dR} / \mathrm{dt})_{40} \\
(\% 02 / \mathrm{min})\end{array}$ & $\begin{array}{l}\text { Grado de } \\
\text { reducción } \\
\text { R60 (\%) }\end{array}$ & $\begin{array}{l}\text { Grado de } \\
\text { metaliza- } \\
\text { ción (\%) }\end{array}$ & ST $\left({ }^{\circ} \mathrm{C}\right)$ & $\mathrm{FT}\left({ }^{\circ} \mathrm{C}\right)$ \\
\hline \multirow{8}{*}{1} & S2 & 35,0 & 66,0 & 1,29 & 73,47 & 68,38 & 1.325 & 1.348 \\
\hline & S4 & 34,1 & 70,0 & 1,37 & 75,50 & 74,94 & 1.312 & 1.338 \\
\hline & S6 & 36,1 & 62,7 & 1,53 & 77,97 & 74,25 & 1.347 & 1.364 \\
\hline & S8 & 38,4 & 67,3 & 1,22 & 71,89 & 78,07 & 1.316 & 1.350 \\
\hline & $\mathrm{S} 10$ & 40,6 & 67,3 & 1,43 & 77,00 & 72,47 & 1.342 & 1.352 \\
\hline & S12 & 43,0 & 72,7 & 1,40 & 77,57 & 73,75 & 1.322 & 1.343 \\
\hline & S14 & 34,7 & 58,0 & 1,37 & 76,72 & 69,96 & 1.336 & 1.352 \\
\hline & S16 & 38,4 & 66,0 & 1,49 & 78,61 & 76,55 & 1.341 & 1.355 \\
\hline \multirow{8}{*}{2} & S18 & 48,9 & 66,7 & 1,56 & 79,10 & 79,70 & 1.329 & 1.350 \\
\hline & $\mathrm{S} 20$ & 45,3 & 70,0 & 1,60 & 86,31 & 80,26 & 1.324 & 1.348 \\
\hline & $\mathrm{S} 22$ & 41,0 & 60,0 & 1,60 & 80,89 & 74,90 & 1.329 & 1.355 \\
\hline & S24 & 45,7 & 64,4 & 1,60 & 82,29 & 80,93 & 1.316 & 1.340 \\
\hline & S26 & 40,9 & 67,3 & 1,40 & 78,23 & 68,43 & 1.352 & 1.366 \\
\hline & S28 & 42,0 & 72,7 & 1,49 & 80,52 & 75,66 & 1.348 & 1.362 \\
\hline & S30 & 47,6 & 58,0 & 1,56 & 81,53 & 80,32 & 1.354 & 1.370 \\
\hline & S32 & 46,9 & 62,7 & 1,46 & 78,80 & 79,07 & 1.341 & 1.355 \\
\hline \multirow{4}{*}{3} & S34 & 32,7 & 70,0 & - & - & - & - & - \\
\hline & S35 & 36,9 & 70,0 & 1,81 & 76,25 & 77,49 & 1.335 & 1.365 \\
\hline & S36 & 35,4 & 70,0 & 1,43 & 76,88 & 77,76 & 1.327 & 1.358 \\
\hline & S37 & 34,6 & 68,7 & 1,60 & 80,10 & 77,35 & 1.321 & 1.351 \\
\hline \multirow{5}{*}{4} & S38 & 40,4 & 66,7 & 1,64 & 82,24 & 77,08 & 1.334 & 1.363 \\
\hline & S39 & 41,2 & 70,6 & - & - & - & - & - \\
\hline & S40 & 40,2 & 68,7 & 1,56 & 78,22 & 81,78 & 1.329 & 1.357 \\
\hline & S41 & 41,2 & 67,3 & - & - & - & - & - \\
\hline & S42 & 40,5 & 68,7 & 1,64 & 80,23 & 78,05 & 1.327 & 1.349 \\
\hline
\end{tabular}


la reducción de hematita a magnetita, acompañada de un aumento de volumen dando lugar a la presencia de tensiones estructurales en el sinterizado ${ }^{[9]}$.

\subsection{Ensayo Tumbler}

La resistencia en frío del sinterizado se determina por el ensayo Tumbler ${ }^{[10]}$ y depende de las resistencias de los componentes minerales individuales, la resistencia del constituyente matriz enlazante y la composición del mineral. El estudio de la resistencia a la fractura de varias fases minerales ha dado lugar al orden siguiente: hematita primaria (o residual) $>$ hematita secundaria (o precipitada) $>$ magnetita $>$ ferritos.

En la tabla IX, se puede observar que los sinterizados presentan buenos resultados para el índice Tumbler, que en algunos casos es superior al $70 \%$.

\subsection{Ensayo de reductibilidad}

La reductibilidad del sinterizado se determina mediante el ensayo ISO que realiza a $900{ }^{\circ} \mathrm{C}^{[5]}$. Se han llevado a cabo investigaciones con el fin de aumentar la reductibilidad del sinterizado, lo que ocasiona una mejora en la estabilidad y un ahorro en el consumo de coque en el horno alto.

En la tabla IX, se puede ver que los sinterizados presentan un buen comportamiento en la reducción, con valores que se encuentran en el intervalo 72-86 \%.

\subsection{Ensayo de temperaturas de reblandecimiento y fusión}

Se han determinado las temperaturas de reblandecimiento y fusión para cada sinterizado fabricado, utilizando un equipo LECO AF-600 según la práctica establecida en el apartado 2.2.5.

Los sinterizados presentan temperaturas de fusión en el intervalo de $1.338-1.370{ }^{\circ} \mathrm{C}$. Las diferencias entre las temperaturas de fusión y reblandecimiento son pequeñas $\left(<35^{\circ} \mathrm{C}\right)$, y la zona cohesiva en el horno alto que se consiga será, por tanto, estrecha, proporcionando de esta forma una buena marcha del horno (Tabla IX).

\subsection{Caracterización de la estructura por microscopia electrónica}

Dada la diversidad de componentes mineralógicos que forman parte de la mezcla de carga mineral, así como de su propia heterogeneidad, se comprende que

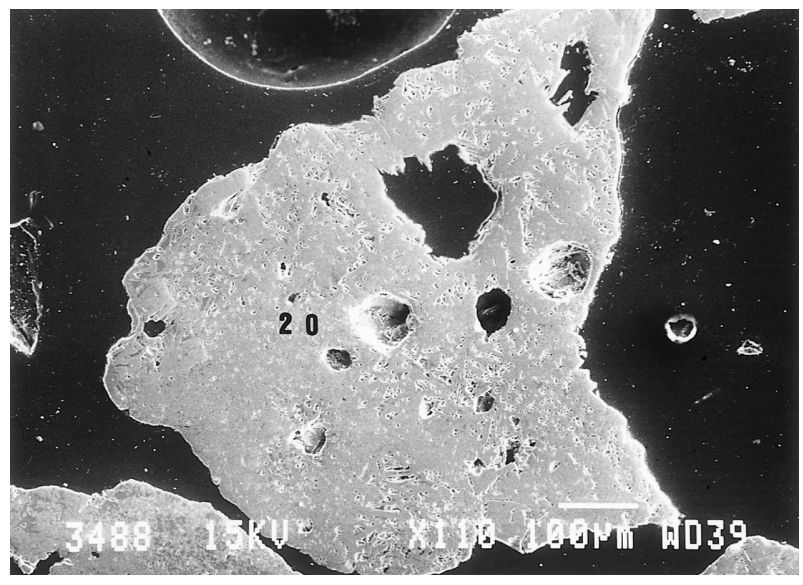

Figura 6. Particula localizada en una muestra del sínter S8 (x110).

Figure 6. Particle located in a sinter simple S8 (x110).

la estructura del sinterizado sea también compleja, estando formada, principalmente, por granos de óxido de hierro y de ferrito cálcico ligados por una matriz de ganga ${ }^{[11-13]}$. Los ferritos, cuya cantidad aumenta con el índice de basicidad, son de fácil reducción y, por aumentar la resistencia mecánica del sínter, hasta ciertos niveles, se consideran constituyentes muy beneficiosos para la carga. La ganga está constituida por silicatos de calcio, hierro y magnesio, difíciles de reducir, y en el horno alto pasan a formar parte de la escoria.

Los sinterizados se han estudiado en un microscopio electrónico de barrido con microsonda JEOL, modelo JSM-840, con un analizador LINK AN10000. Se realiza una exploración de las partículas de cada muestra de sinterizado. Se realiza una serie de fotos de aspectos generales y en detalle de las muestras y en diferentes partículas se ha realizado el análisis cuantitativo puntual en una o en varias fases localizadas en la partícula.

La figura 6 corresponde a una partícula elegida al azar del sínter S8. La partícula es de naturaleza muy heterogénea, como lo son en general todas las partículas de los sinterizados. Esta partícula ha sido analizada en dos zonas. En el borde izquierdo existen las fases muy entremezcladas de hematita, ferritos aciculares y columnares, ganga y presencia de un poro relativamente grande (Fig. 7). En una zona del centro hay presencia de hematita precipitada y magnetita precipitada sobre una matriz de ferritos (Fig. 8). En la tabla $\mathrm{X}$ se ofrece el análisis químico puntual de las fases identificadas en la partícula. El contenido en oxígeno se calcula por diferencia hasta 100 de la suma de los demás elementos. 


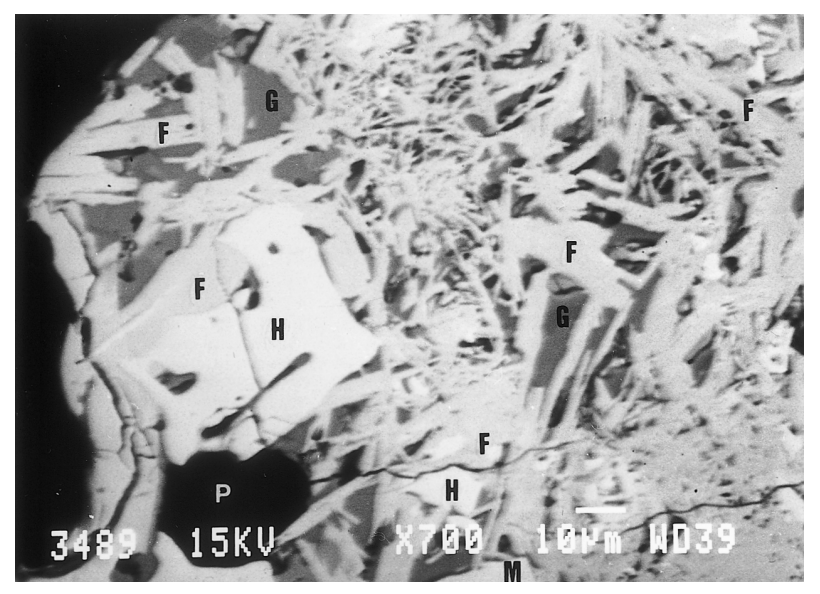

Figura 7. Detalle del borde izquierdo de la partícula mostrada en la figura 6 (x700). $\mathrm{H}=$ hema-

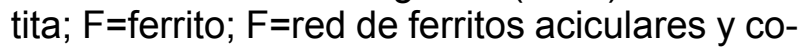
lumnares; $G=$ ganga; $P=$ poro.

Figure 7. Close view of the left edge of the particle shown in fugure 6 (x700). $H=$ hematite; $F=$ ferrite; $F=$ network of acicular and columnar ferrites; $G=$ Ganga; $P=$ pore.

\section{DISCUSIÓN}

\subsection{Sinterización de mezclas minerales}

El sínter es la principal mezcla mineral que contiene minerales de hierro que se utiliza como material de carga en el horno alto. Su calidad es muy importante para la buena marcha del horno ${ }^{[14]}$.

Las propiedades del fundido que se forma durante el proceso de sinterización determinan la estructura de las fases ligantes que se originan en el sinterizado. Las propiedades del fundido, momentos antes de la solidificación, dependen, en gran medida, de la

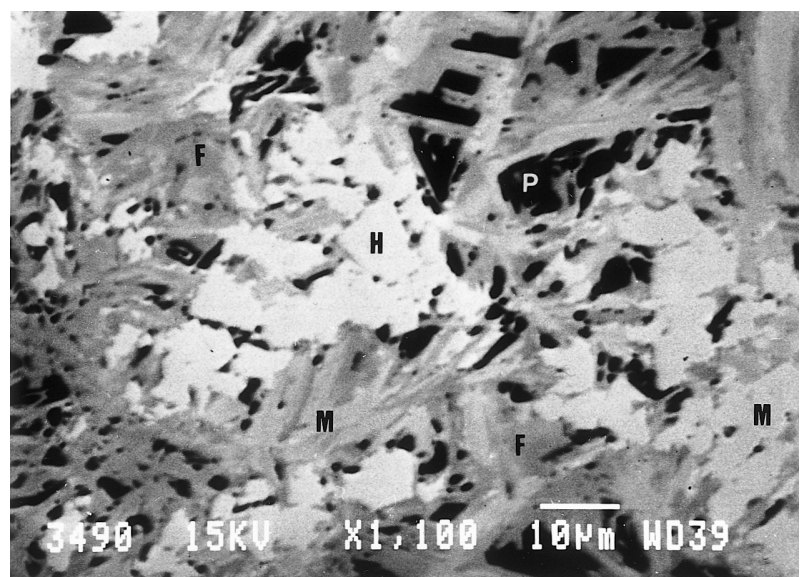

Figura 8. Detalle de una zona central de la partícula mostrada en la figura 6 (x1100). $\mathrm{H}=$ hematita; $M=$ =magnetita; $F=$ ferrito; $P=$ poro.

Figure 8. Close view of the central area of the particle shown in figure $6(x 1100) . H=$ hematite; $M=$ magnetite; $F=$ ferrite $;=$ pore.

composición química de la capa de finos adherentes a los gránulos y del comportamiento frente a la asimilación de las partículas del núcleo ${ }^{[15-18]}$.

Los primeros trabajos sobre la estructura de las mezclas minerales granuladas se realizaron por investigadores de la Nippon Steel Corporation (NSC) y fue definido el término de cuasipartícula ${ }^{[19-21]}$.

La cuasipartícula está formada por el núcleo de hematita que, durante la sinterización, permanece sin fundir, rodeado por granos más finos de mineral con ganga de sílice y en presencia de una basicidad $\left(\mathrm{CaO} / \mathrm{SiO}_{2}\right)$ alta. Las partículas mayores de $0,7 \mathrm{~mm}$ actúan como núcleos y las partículas inferiores a 0,2 $\mathrm{mm}$ actúan como finos adherentes. Las partículas intermedias son difíciles de granular y no tienen un papel bien definido.

Tabla X. Análisis químico puntual de las fases identificadas en una partícula del sínter S8, $\%$ en masa

Table X. Point chemical analysis of the identified phases of particle of sínter S8, \% mass

\begin{tabular}{ccccccccc}
\hline Partícula & Fase & Fe & Ca & Al & Si & Mn & Mg & O \\
\hline \multirow{4}{*}{ Borde izquierdo } & Hematita & 66,26 & & 0,46 & 0,13 & & & 33,18 \\
& Ferrito & 51,28 & 8,32 & 1,34 & 2,46 & 0,28 & 0,12 & 36,20 \\
& Ganga & 2,99 & 29,34 & 0,06 & 14,97 & & & 52,64 \\
\hline \multirow{4}{*}{ Zona centro } & Hematita & 68,53 & & & & & & 31,47 \\
& Magnetita & 66,61 & 1,25 & & & 0,88 & & 31,26 \\
& Ferrito & 51,81 & 8,26 & 1,01 & 2,67 & & & 36,25 \\
\hline
\end{tabular}


La capa adherente al núcleo, durante la sinterización, experimenta la formación de ferritos por reacción sólido-líquido entre la hematita y un fundido de $\mathrm{Fe}_{2} \mathrm{O}_{3} \cdot \mathrm{CaO}$ que contiene pequeñas cantidades de $\mathrm{SiO}_{2}$ y $\mathrm{Al}_{2} \mathrm{O}_{3}$.

Se considera como estructura óptima para la reductibilidad del sinterizado en el horno alto, la formada por un núcleo de hematita primaria (sin fundir) rodeada de ferrito acicular. Esta estructura se puede conseguir con una granulación adecuada de la mezcla mineral y realizando la sinterización a temperatura inferior a $1.300{ }^{\circ} \mathrm{C}$ y se producen las reacciones siguientes: en el lecho de las cuasipartículas se genera, a unos $1.200^{\circ} \mathrm{C}$, un fundido (que consiste, principalmente, en $\mathrm{Fe}_{2} \mathrm{O}_{3}$ y $\mathrm{CaO}$ ); el óxido de hierro y las partículas minerales finas se asimilan en el fundido. Si el fundido penetra en los granos de hematita del núcleo se produce una rotura interfacial, quedando una hematita primaria (sin fundir) que se considera beneficiosa para el sinterizado porque mejora el índice de reductibilidad (RI). Cuando $\mathrm{CaO}$ y $\mathrm{Al}_{2} \mathrm{O}_{3}$ se asimilan en el fundido, éste reacciona con el óxido de hierro y genera ferrito cálcico acicular (de tamaño $<10 \mu \mathrm{m}$ ) conteniendo $\mathrm{Al}_{2} \mathrm{O}_{3}$ y $\mathrm{SiO}_{2}$ como disoluciones sólidas, según la reacción global:

$$
\begin{gathered}
\mathrm{Fe}_{2} \mathrm{O}_{3}+\mathrm{CaO}+\mathrm{SiO}_{2}+\mathrm{Al}_{2} \mathrm{O}_{3} \rightarrow \\
\mathrm{SiO}_{2} \cdot \mathrm{CaO} \cdot(\mathrm{Fe}, \mathrm{Al})_{2} \mathrm{O}_{3}
\end{gathered}
$$

Estos silicoferritos de calcio y aluminio (SFCA) se consideran componentes muy beneficiosos en la estructura del sinterizado puesto que poseen buena reductibilidad y aportan resistencia mecánica al sinterizado mejorando el índice Tumbler (TI). Al sinterizar a baja temperatura disminuye la formación de magnetita (menor $\mathrm{FeO}$ ) y el sinterizado mejora el RI y el índice de degradación con la reducción (RDI) ${ }^{[22-24]}$.

Cuando se sinteriza a temperatura superior a 1.300 ${ }^{\circ} \mathrm{C}$, parte del ferrito se disuelve y funde para convertirse en hematita o magnetita y en componentes de ganga ${ }^{[25-27]}$. Al enfriarse el fundido se forman, como fases nuevas, grandes cristales de ferrito que se reducen peor que el ferrito acicular, y hematita precipitada, también llamada hematita secundaria, que es perjudicial para el RDI ${ }^{[22]}$.

\section{2. Índice RDI}

El índice RDI es un parámetro muy importante que se utiliza como referencia en todos los trabajos de sinterización y sirve para conocer cómo será el comportamiento del sinterizado a la degradación, a baja temperatura en el horno alto, en la zona baja de la cuba.

El índice RDI tiene el valor más bajo (es el mejor) para la mezcla mineral 3 (Tabla XI) y, esto, se puede asociar con el menor contenido en $\mathrm{Al}_{2} \mathrm{O}_{3}$ de todas las mezclas $\left(1,02 \% \mathrm{Al}_{2} \mathrm{O}_{3}\right)^{[28-31]}$ y a la presencia de un contenido en $\mathrm{MgO}$ más elevado en la mezcla, que resulta beneficioso para el RDI ${ }^{[32]}$.

La mezcla 2 tiene los valores más elevados para este índice, lo que hace suponer que pueda ser causa inherente a la composición de la mezcla utilizada.

\section{3. Índice Tumber}

Este ensayo permite determinar la reducción de tamaño por impacto y abrasión de los sinterizados en su manipulación, transporte y en el proceso del horno alto.

La resistencia mecánica en frío está directamente relacionada con la tendencia a la formación de finos en el transporte y manipulación desde la máquina de sinterización hasta el tragante del horno. Se han publicado diversos trabajos sobre los factores que influyen en la resistencia de los sinterizados ${ }^{[33-37]}$.

Los mejores sinterizados, desde le punto de vista de su resistencia, fueron conseguidos para las mezclas 1 y 2, con un índice Tumbler superior al 72 \% (Tabla XI).

\section{4. Índice de reductibilidad}

La reductibilidad es una característica importante de los sinterizados que mide la facilidad de ceder oxígeno durante la reducción en la cuba del horno alto, dando una idea de la necesidad del consumo de combustible en el horno. La porosidad y la estructura de los sinterizados y sus fases minerales están íntimamente relacionados con la reductibilidad de éstos. Una estructura heterogénea es más reductible que una estructura homogénea ${ }^{[38-40]}$. También es posible predecir el comportamiento a la reductibilidad con la concentración de cada fase presente ${ }^{[41]}$.

La reductibilidad de las fases minerales disminuyen en el orden:

$$
\begin{gathered}
\mathrm{Fe}_{2} \mathrm{O}_{3}>\mathrm{CaO} \cdot 2 \mathrm{Fe}_{2} \mathrm{O}_{3}>\mathrm{CaO} \cdot \mathrm{Fe}_{2} \mathrm{O}_{3}> \\
2 \mathrm{CaO} \cdot \mathrm{Fe}_{2} \mathrm{O}_{3}>\mathrm{Fe}_{3} \mathrm{O}_{4}
\end{gathered}
$$

La hematita y magnetita se reducen rápidamente a wustita $(\mathrm{FeO})$, pero las velocidades difieren para la posterior reducción a hierro metálico ${ }^{[42]}$. A partir de la hematita, la wustita se reduce de forma homogénea y rápida, aunque alguna wustita se encuentra rodeada de metal. A partir de la magnetita, la reducción es 
Tabla XI. Análisis químico, índices RDI, Tumbler y de reductibilidad, y temperaturas de reblandecimiento y fusión de los sinterizados fabricados en planta piloto con $60 \mathrm{~cm}$ de altura del lecho, $35 \%$ de finos de retorno y 1.9 de basicidad

Table XI. Chemical analysis, RDI, Tumbler and reductibility indexes, and softening and meting temperatures of sinters manufactured in pilot plant with bed height $60 \mathrm{~cm}$, return fines $35 \%$ and basicity 1,9

\begin{tabular}{|c|c|c|c|c|}
\hline & \multicolumn{4}{|c|}{ Mezcla mineral } \\
\hline & 1 & 2 & 3 & 4 \\
\hline & S12 & S28 & $\begin{array}{c}\text { Valor medio } \\
\text { S34, S35, } \\
\text { S36, S37 }\end{array}$ & $\begin{array}{c}\text { Valor medio } \\
\text { S38, S39, S40, } \\
\text { S41, S42 }\end{array}$ \\
\hline \multicolumn{5}{|l|}{ Análisis: } \\
\hline Fe total, \% & 56,2 & 56,5 & 55,8 & 55,7 \\
\hline $\mathrm{FeO}, \%$ & 4,6 & 5,0 & 4,6 & 4,8 \\
\hline $\mathrm{Al}_{2} \mathrm{O}_{3}, \%$ & 1,15 & 1,10 & 1,02 & 1,25 \\
\hline $\mathrm{MgO}, \%$ & 1,58 & 1,66 & 1,65 & 1,79 \\
\hline $\mathrm{CaO} / \mathrm{SiO}_{2}$ & 1,93 & 1,82 & 1,93 & 1,95 \\
\hline $\mathrm{RDI}(<3 \mathrm{~mm}), \%$ & 43,0 & 42,0 & 36,8 & 40,7 \\
\hline Tumbler (>6.3 mm), \% & 72,7 & 72,7 & 69,1 & 68,4 \\
\hline Grado de reducción $\mathrm{R}_{60}, \%$ & 77,6 & 80,5 & 77,7 & 80,2 \\
\hline Temp. de reblandecimiento (ST), ${ }^{\circ} \mathrm{C}$ & 1.322 & 1.348 & 1.328 & 1.330 \\
\hline Temp. de fusión (FT), ${ }^{\circ} \mathrm{C}$ & 1.343 & 1.362 & 1.358 & 1.356 \\
\hline
\end{tabular}

topoquímica, según la secuencia $\mathrm{Fe}_{3} \mathrm{O}_{4}$ à $\mathrm{FeO}$ à $\mathrm{Fe}, \mathrm{y}$ casi todos los granos de magnetita son rodeados de hierro metálico, que retarda la reducción posterior.

La reductibilidad de SFCA se puede relacionar con su morfología, su porosidad y si está recubierto de vidrio ${ }^{[43-45]}$. El ferrito acicular $(<10 \mu \mathrm{m})$ formado a baja temperatura $\left(<1.300^{\circ} \mathrm{C}\right)$, es más reductible, mientras que el ferrito columnar $(>10 \mu \mathrm{m})$ formado a alta temperatura $\left(>1.300^{\circ} \mathrm{C}\right.$, posiblemente recubierto de vidrio) es menos reductible. La hematita primaria es más reductible que la hematita secundaria, a causa de su porosidad intrínseca.

En general para los sinterizados fabricados con las mezclas minerales 1 y 2 , el grado de reducción aumenta a medida que la basicidad crece de 1,6 a1,9, debido a que a mayor basicidad aumenta el contenido de ferritos y, como consecuencia, existirá una mayor reductibilidad.

La mayor reductibilidad que presentan los sinterizados de la mezcla 2 respecto a los sinterizados de la mezcla 1 (Tabla XI), se puede explicar como una mayor proporción de minerales goetíticos en la mezcla 2 , ya que éstos son más porosos y por tanto más reductibles, hecho que se corresponde con investigaciones en las que se observó que los poros finos producidos durante la reducción conducen a un aumento de la porosidad y, con ello, a mayores reductibilidades.

La mejor reductibilidad de los sinterizados de la mezcla 4 respecto a la mezcla 3 , se puede explicar de forma análoga (Tablas IV y XI)

\subsection{Reblandecimiento y fusión}

El funcionamiento de un horno alto está condicionado por la geometría y situación de la zona cohesiva que está delimitada por las isotermas de reblandecimiento y fusión. ${ }^{[48-50]}$ La zona cohesiva está constituida por capas alternativas de sinterizado en estado pastoso y coque. Son, estas últimas, denominadas "ventanas de coque", las que permiten el paso del gas reductor hacia las zonas superiores del horno. Es por tanto importante que la zona cohesiva sea lo más estrecha posible para facilitar el paso del gas y que esté lo más bajo posible en el horno alto para que la zona de preparación del horno situada encima de la zona cohesiva tenga extensión suficiente para permitir la reducción de los óxidos de hierro. Para conseguir ambos condicionantes, es necesario que las temperaturas ST y FT sean lo más altas posibles y de diferencia mínima. Esto se ha conseguido con los sinterizados fabricados (Tabla XI). 


\subsection{Estructura de los sinterizados}

Se ha estudiado, por microscopia electrónica, la estructura y composición de una serie de muestras de sinterizados. Se ha detectado la presencia de las fases hematita primaria (no asimilada o residual), hematita secundaria (precipitada), magnetita primaria (no asimilada o residual), magnetita secundaria (precipitada) y ferritos como componentes mayoritarios y ganga en menor cantidad. Hay presencia de porosidad, con microporos en muchos casos, que favorece la reductibilidad del sínter. La estructura es siempre muy heterogénea con las fases muy entremezcladas.

Los ferritos analizados son del tipo SFCA (silicoferritos de calcio y aluminio), que se forman por reacción sólido-líquido entre la hematita y un fundido de $\mathrm{Fe}_{2} \mathrm{O}_{3} \cdot \mathrm{CaO}$, con posterior asimilación de $\mathrm{SiO}_{2}$ y $\mathrm{Al}_{2} \mathrm{O}_{3}$ a este fundido. La fórmula química del SFCA puede ser escrita como $5 \mathrm{CaO} \cdot 2 \mathrm{SiO}_{2} \cdot 9(\mathrm{Fe}, \mathrm{Al})_{2} \mathrm{O}_{3}{ }^{[51}$ y ${ }^{52]}$. Estos ferritos son beneficiosos para la estructura del sínter porque mejoran la resistencia y reductibilidad. Se ha detectado, en numerosos casos, la presencia de ferritos aciculares, que se consideran como los que ofrecen condiciones óptimas. La ley, en hierro, de los ferritos es mayor para los sinterizados de basicidad 1,9 que para la basicidad 1,6.

En algunos sinterizados se ha detectado la estructura óptima para la reductilidad, formada por un núcleo de hematita primaria (residual) rodeado por una red de ferritos aciculares (Fig. 9).

\section{CONCLUSIONES}

Se han caracterizado una serie de minerales de hierro, fundentes y coque. Se han tratado en plata piloto distintas mezclas de minerales de hierro de diferente procedencia, utilizando distintos parámetros de operación para establecer las condiciones mejores de fabricación de los sinterizados. Los resultados obtenidos en planta piloto, para la obtención de sinterizados y en laboratorio, para la caracterización de sus propiedades físicas y estructurales, permiten establecer las siguientes conclusiones:

- Se han obtenido sinterizados de buenas características, adecuados para una marcha óptima del horno alto. Se ha logrado la fabricación de estos sinterizados con consumos energéticos reducidos, especialmente, bajo consumo de coque.

- En general, los sinterizados tienen una estructura con presencia de ferritos que aportan propiedades beneficiosas para la resistencia y reductibilidad del sínter. Se ha detectado la estructura óptima formada por un núcleo de hematita rodeada por una red de ferritos aciculares. Esta es-

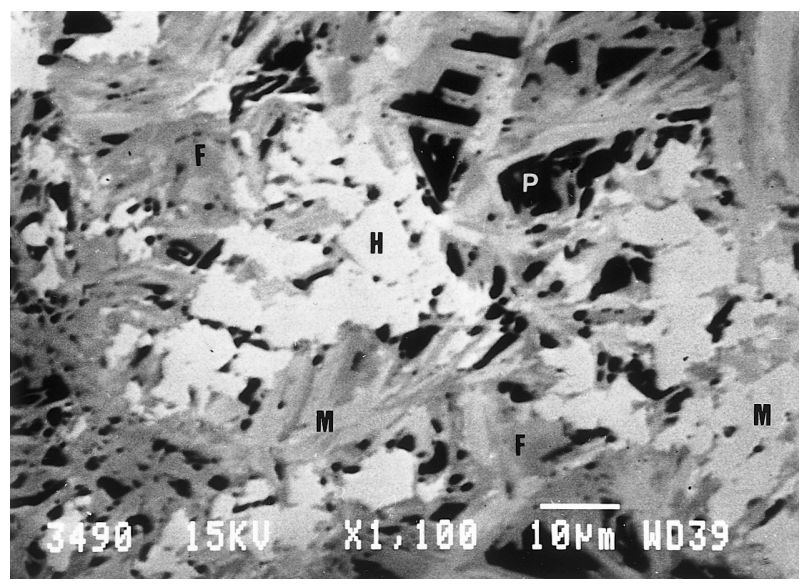

Figura 9. Estructura óptima de sinterizado formada por un núcleo de hematita primaria rodeada por una red de ferritos aciculares $(x 750) \mathrm{H}=$ hematita; $\mathrm{F}=$ red de ferritos. Presencia de ganga (oscuro) y poros (negro).

Figure 9. Optimized sintered structure formed by a nucleus of primary hematite surrounded by a network of acicular ferrites (x750) $\mathrm{H}=$ hematite; $F=$ ferrite network. Presence of (dark) ganga and (black) pores.

tructura se ve favorecida al trabajar con basicidades más altas.

- Los sinterizados fabricados con mayor altura de capa, presentan mejor comportamiento a altas temperaturas, siendo éstas más elevadas, tanto en el inicio de reblandecimiento como en la fusión, a la vez que la diferencial entre ambas temperaturas es más pequeña. Este mejor comportamiento será beneficioso para una posición más favorable de la zona cohesiva en el horno alto.

- En base a los resultados obtenidos se ha definido una ley racional de composición de mezclas minerales, que permite una mejora de la productividad en la fabricación de los sinterizados.

\section{REFERENCIAS}

[1] T. Haga, A. Oshio, D. Shibata, S. Kasama, T. Kozono y Y. Hida, 4th ECIC, Paris, 2000 ATSRM, pp. 118-125.

[2] A. Formoso, A. Moro, G. Fernández-Pello, M. Muñiz, J. Jiménez, A. Moro y A. Cores, Rev. Metal. Madrid 36 (2000) 244-265.

[3] A. Formoso, A. Moro, G. Fernández-Pello, J.L. Menéndez, M. Muñiz y A. Cores, Ironmaking Steelmaking 30 (2003) 447-460. 
[4] V. Errigo, M. Pintin, y C. Pignatelli, 3rd Int. Symp. on Agglomeration. Nuremberg Alemania, 1981, pp. A160-A174.

[5] Fifth Draft ISO Proposal, ISO/TC-120/SC-2/284E, ISO, Geneve, Suiza, 1974.

[6] D1857-68 (Reapproved 1980), ASTM, Pittsburgh, PA (EE.UU.), 1980.

[7] Third draft, ISO Proposal, ISO/TC-102/SC3/285 E, 1974.

[8] H. A. Kortman y A. Burghardt, Agglomeration 77, Ed. Sastry, K.V.S., AIME, New York, EE.UU., 1977, pp. 219-231.

[9] P.W. Roller, Trans. ISIJ. 26 (1986) 834-835.

[10] E 279-69 (Reapproved 1979).

[11] S.N. Ahsan, T. Mukherjee y J. A. Whiteman, Ironmaking Steelmaking 10 (1983) 54-64.

[12] T. Mukherjee y J.A. Whiteman, Ironmaking Steelmaking 12 (1985) 151-155.

[13] G.O. Egundebi, J.A. Whiteman, Ironmaking Steelmaking 16 (1989) 379-385.

[14] C.E. Loo, ICSTI Ironmaking Conf. Proc., 1998, pp 1.299-1.316.

[15] Li. Heng Hsieh y J.A. Whiteman, ISIJ Int. 33 (1993) 462-473.

[16] A. Napoleão, P. Pinheiro, L. Caporali, D.L.A. Oliveira, L. Fujikawa y R. Vervenne, 3rd Int. Conf. on Sci. Tech. Ironmaking Proc., Düsseldorf, Alemania, 2003, pp. 127-132.

[17] D. Debrincat, C.E. Loo y M. F. Hutchens, ISIJ Int. 44 (2004) 1.308-1.317.

[18] A. Cores, A. Babich, M. Muñiz, A. Isidro y S. Ferreira, Ironmaking Steelmaking 34 (2007) 231-240

[19] T. Fuori, K. Sugawara, M. Kagawa, S. Uno, M. Kamazu, T. Fujiwara y A. Sawamura, Nippon Steel Tech. Rep. Overseas 10 (1997) 36-46.

[20] Y. Hida, M. Sasaki, K. Sato, M. Kagawa, T. Miyazaki, H. Soma, H. Naito y M. Taniguchi, Nippon Steel Tech. Rep. Overseas 35 (1987) 59-67.

[21] S. Wu, E. Kasai y Y. Omori, Proc. 6th Int. Iron Steel Cong., Nagoya, Japón, Iron and Steel Inst. of Japan, 1990, pp. 15-22.

[22] Y. Ishikawa, Y. Shimomura, M. Sasaki, Y. Hida y H. Toda, ISS Ironmaking Conf. Proc. 42 (1983) $17-29$.

[23] H. Toda, T. Senzaki, S. Isozaki y K. Kato, Trans. Iron Steel Inst. Jpn, 24 (1984) 187-196.

[24] P. R. Dawson, Proc. 4th Int. Symp. Agglomeration, Ed. C.E. Capers. Iron and Steel Society of AIME, Warrendale, PA, EE.UU. 1985, pp. 243-250.

[25] F. Matsuno y T. Harada, Trans. ISIJ 21 (1981) 318-325.

[26] Li.- H. Hsieh y J. A. Whiteman, ISIJ Int. 29 (1989) 625-634.

[27] Li.- H. Hsieh y J. A. Whiteman, ISIJ Int. 29 (1989) 24-32.
[28] I. Shigaki, M. Sawada, M. Maekawa y K. Narita, Trans. ISIJ 22 (1982) 838-884.

[29] I. Shigaki, M. Sawada, y N. Gennai, Trans. Iron Steel Inst. Jpn. 26 (1986) 503-511.

[30] F. Matsuno, S. Nishikida y H. Ikesaki, Trans. Iron Steel Inst. Jpn. 24 (1984) 1.040-1.049.

[31] F. Matsuno, S. Nishikida y H. Ikesaki, Trans. Iron Steel Inst. Jpn. 25 (1985) 953-962.

[32] Y. Yamaoka, S. Nagaoka, Y. Yamada y R. Ando, Trans. Iron Steel Inst. Jpn. 14 (1974) 185-194.

[33] D. Jeulin, Rev. Métall. Cah. Inf. Tech. 78 (1981) 481-489.

[34] E. Kasai, S. Wu y Y. Omori, ISIJ Int. 31 (1991) 17-23.

[35] E. Kasai, W. J. Ranking, R. R. Lovel y Y. Omori, ISIJ. Int. 29 (1989) 641-653

[36] R. G. Lyons y W. J. Davies, ISS Ironmaking Conf. Proc. 47 (1988) 647-656.

[37] J. Okazaki, K. Higuchi, S. Nomura, M. Nakano y M. Naito, 3rd Int. Conf. Sci. Tech. Ironmaking Proc., Düsseldorf, Alemania, 2003, pp. 123 126.

[38] Y. Ishikawa, Y. Shimomura, M. Sasaki, Y. Hida y H. Toda, ISS Ironmaking Conf. Proc. 42 (1983) 17-29.

[39] H. Toda, T. Senzaki, S. Isozaki y K. Kato, Trans. ISIJ 24 (1984) 187-196.

[40] S.C. Panigrahy, M.A.J. Rigaud y G.S. Hegedus, Ironmaking Steelmaking 21 (1994) 353-631.

[41] N. Sakamato, H. Fukuyo, Y. Iwata y T. Niyashita, Proc. 12th Mc Master Symp, Hamilton ON, Canadá, Mc Master University, 1984, pp. 137-150.

[42] T. Maeda y T. Ono, Trans. ISIJ 25 (1985) 1.191 1.193.

[43] S. Wang, W. Gao y L. Kong, Ironmaking Steelmaking 25 (1998) 296-301.

[44] T. Maeda, K. Nishioka, K. Nakashima y M. Shimizu, ISIJ Int. 44 (2004) 2046-2051.

[45] N.V.Y. Scarlett, M.I. Pownceby, I.C. Madsen y A.N. Christensen, Metall. Mat. Trans. B, 35 (2004) 929-936.

[46] N. J. Bristow y A. G. Waters, Trans. Inst. Min. Metall. 100 (1991) C1-C10.

[47] N. J. Bristow, J. Gross y A. G. Waters, Iron Steelmaker (1991) 61-70

[48] M.A. Propster y J. Szekely, Ironmaking Steelmaking 6 (1979) 209-220.

[49] S. Wakamaya, Y. Kanamaya y Y. Okuno, Ironmaking Steelmaking 6 (1979) 261-267.

[50] N.J. Busby, T.A.T. Fray y D.C. Goldring, Ironmaking Steelmaking 21 (1994) 229-236.

[51] JCPDS (EE.UU.), (1983) 33-250.

[52] P. R. Dawson, Ironmaking Steelmaking 20 (1993) 137-143. 